\title{
Multilength-Scale Modeling: Crystal-Plasticity Models in Implicit Finite Element Codes
}

David D. Sam

Wayne E. King

RECEIVES

JUL 08 1996

OSTI

March 1996

This is an informal report intended primarily for internal or limited external listribution. The opinions and conclusions stated are trose of the author and may If may not be those of the Laboratory.

Nork periormed under the auspices of the U.S. Departoent of Energy by the aprence Livermore Tiational Laboratory under Contract W-7-405-ENG-48. 


\section{DISCLATMER}

This document was prepared as an account of work sponsored by an agency of the United States Government. Neither the United States Government nor the University of California nor any of their employees, makes any warranty, express or implied, or assumes any legal liability or responsibility for the accuracy, completeness, or usefulness of any information, apparatus, product, or process disclosed, or represents that its use would not infringe privately owned rights. Reference herein to any specific commercial products, process, or service by trade name, trademark, manufacturer, or otherwise, does not necessarily constitute or imply its endorsement, recommendation, or favoring by the United States Government or the University of California. The views and opinions of authors expressed herein do not necessarily state or reflect those of the United States Government or the University of California, and shall not be used for advertising or product endorsement purposes.

This report has been reproduced directly from the best available copy.

Available to DOE and DOE contractors from the Office of Scientific and Technical Information

P.O. Box 62, Oak Ridge, TN 37831

Prices available from (615) 576-8401, FTS 626-8401

Available to the public from the

National Technical Information Service

U.S. Department of Commerce

5285 Port Royal Rd.,

Springfield, VA 22161 


\title{
Multilength-scale Modeling: Crystal-Plasticity Models in Implicit Finite Element Codes
}

\author{
David D. Sam* and Wayne E. King** \\ *New Technologies Engineering Division, Mechanical Engineering \\ **Materials Science and Technology Division, Chemistry and Materials Science Directorate
}

A multidisciplinary team of researchers from Chemistry \& Materials Science and Mechanical Engineering have recently completed the first year of an LDRD Director's Initiative. This initiative involves the development of physics-based multilength-scale models to optimize casting and deformation processing of polycrystalline materials. The purpose of this initiative is to develop models to simulate the material's mechanical response on the meso length-scale. The meso length-scale refers to the realm where the material microstructure (e.g., grain size and shape, crystallite orientation, etc.) is resolved but dislocations are homogenized. The recent development of two experimental technologies has provided a unique capability to validate material models on the mesolength-scale. The first of these technologies is the ultrahigh-vacuum diffusion bonding machine ${ }^{1}$. This machine has been demonstrated to build bicrystals with high-precision planar grain boundaries joining two precisely oriented crystallites. The second technology is Orientation Imaging Microscopy ${ }^{2}$ (OIM). This technology has been demonstrated to precisely determine the local lattice orientation in a microstructure. Hence, this provides a powerful tool to characterize local lattice rotations following specified levels of deformation. The coupling of these two technologies provides a tool for detailed study of a material's mechanical response due to the presence of an interface or grain boundary.

The first phase of this initiative, as described here, was to build a bicrystal consisting of two crystallites joined by a single, planar grain boundary. The bicrystal was then deformed by channel-die compression while recording the deformation response. Comparisons were made between the measured response with predictions using a three-dimensional finite element simulation that uses a crystalplastic material model.

In this study, we are primarily interested in the deformation response of crystalline solids composed of face-centered cubic (fcc) crystallites. For an fcc system the deformation is assumed to result solely from shear slipping along close-packed crystallographic planes. By inspection of an fcc unit cell, it is seen that it has 4 unique slip planes, each having 3 unique slip directions (not counting their negatives). A slip plane together with an associated slip direction constitutes a slip system. Thus, there are a total of 12 slip systems that may operate in the fcc single crystal to achieve a deformation. An arbitrary deformation in a single crystal is achieved by the composite effect of all contributing (active) slip systems.

One objective of this work was to incorporate a tensor-based single crystal constitutive model that includes the kinematics of the crystal's deformation response coupled with a scalar law that relates the shear stress, resolved onto the slip plane and slip direction, with the associated slip rate. From the 
description of the crystal's deformation response, to achieve plastic slipping on a given slip system the yield criterion must be satisfied according to Schmid's law, which states that plastic slipping initiates when the shear stress, resolved onto a slip system, reaches a critical value.

The constitutive model that was applied falls within the general framework of the finite deformation theory of crystals that has been thoroughly documented in the literature. It assumes that the elastic response of the crystallite is undisturbed by plastic slip and hence, the plastic deformation of a crystal may then be envisioned as a decomposition into two subsequent deformations: first a homogeneous shearing on a given slip system, followed by the simultaneous rigid body rotation and elastic distortion of the lattice. The kinematic description of the crystal mechanics used in the constitutive model follows that presented by Peirce, Asaro and Needleman ${ }^{3}$ and later adapted for use in the finite deformation code, ABAQUS, by Becker ${ }^{4}$. The model was then adapted for use in LLNL's implicit finite deformation code, NIKE3D.

MODELING: To illustrate the behavior of this material model, a finite element model was produced for a bicrystal with a grain boundary misorientation specified to induce strain localization near the interface. This was done by selecting respective crystal orientations such that one crystallite would be more resistant to plastic slipping than the other crystallite, thus illustrating strong inhomogeneity of plastic strain due to the misorientation across the interface. A flat planar surface was modeled as the interface between the two crystallites. Each crystallite in the bicrystal was modeled with a
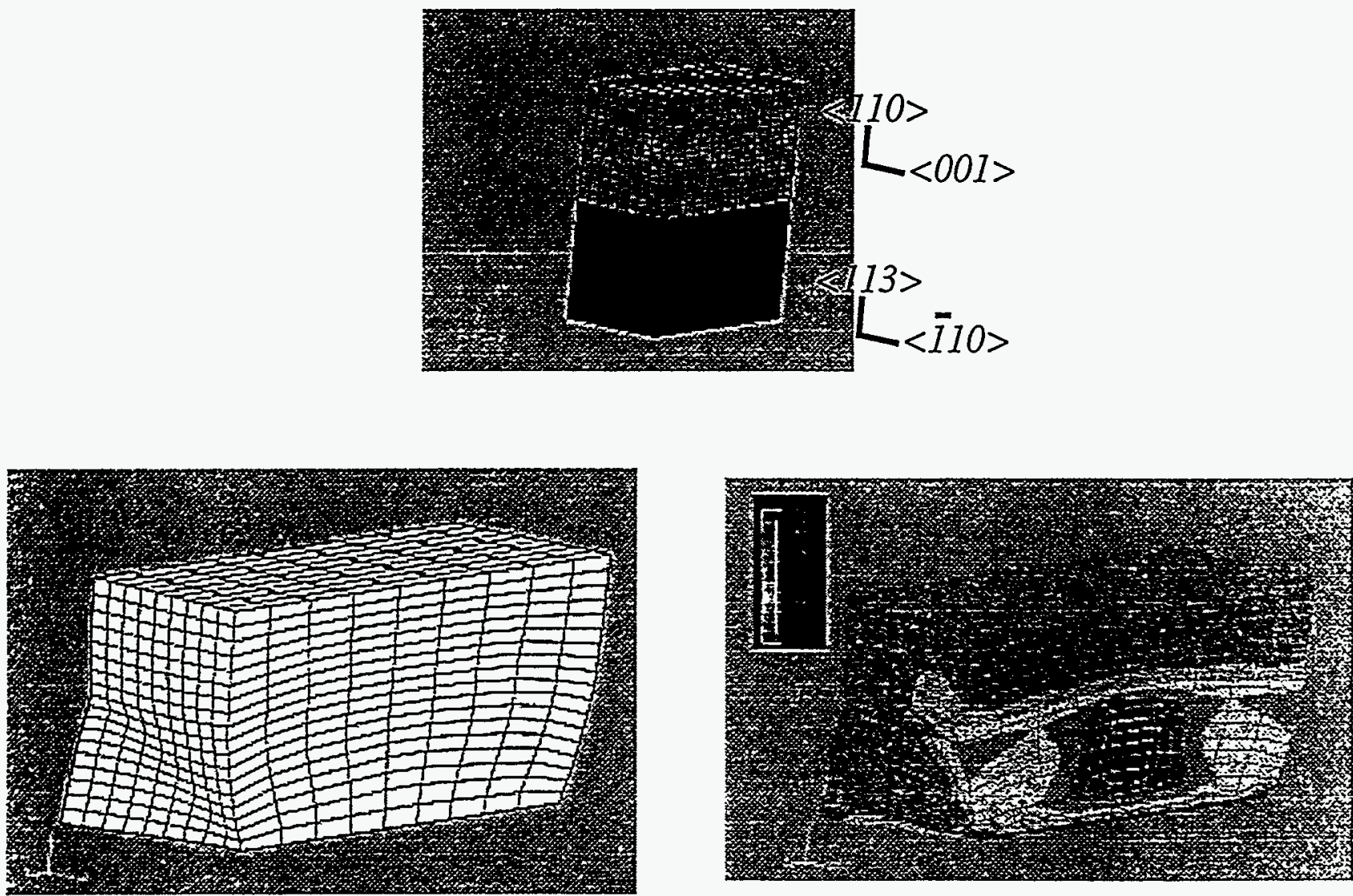

Figure 1. Undeformed bicrystal mesh (top), deformed mesh (left), deformed mesh with strain contours (right). 
$9 \times 9 \times 9$ grid that resulted in 1458 total elements. The deformation was then modeled as a channel-die compression operation by deforming the bicrystal in the direction normal to the grain boundary interface, constraining it along a transverse dimension and allowing free plastic flow in the third dimension. The simulation was carried out to $30 \%$ bulk deformation along compression direction. The predicted deformation was then stored for comparison with the experiments. During the simulation, lattice deformation history was dumped to a file from which, using polar decomposition, the predicted lattice rotations were obtained as function of grid location. From this information, simulated pole figures were generated for comparison with the experiments (see Fig. 1).

EXPERIMENTS: Using the ultra high vacuum diffusion bonding machine with high purity

Compression Direction

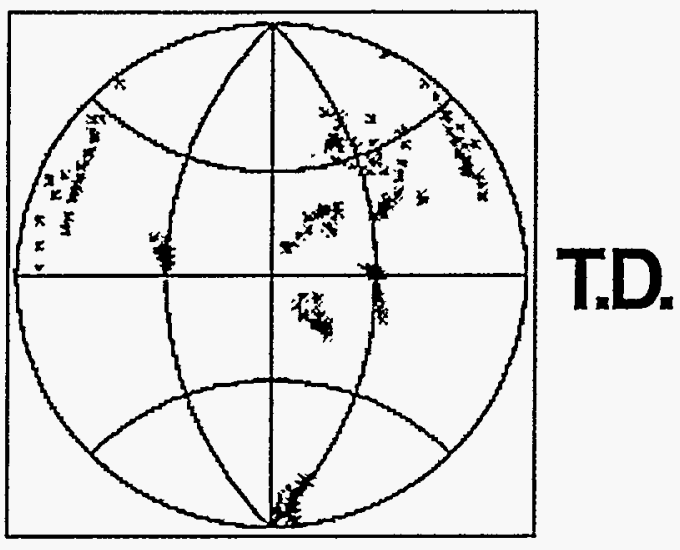

\section{(Channel Direction}

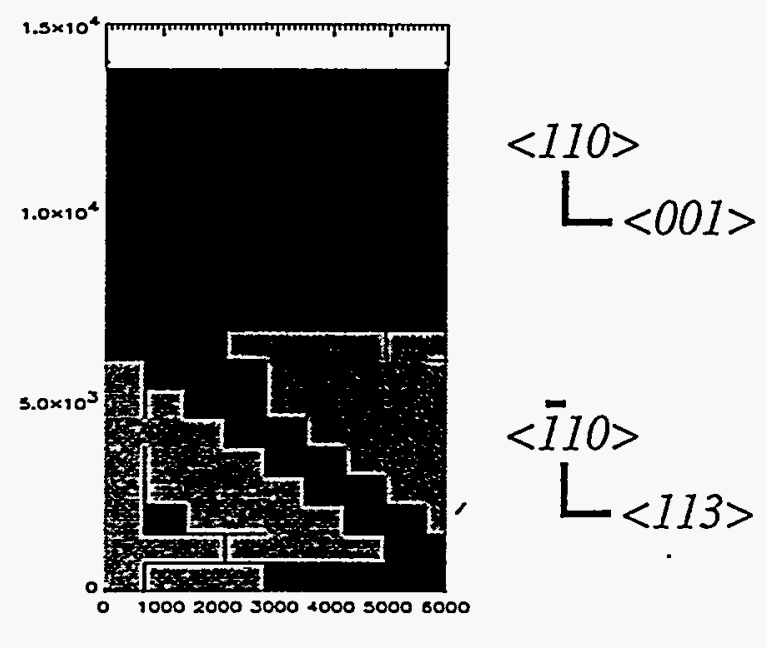

Figure 2. Simulated pole figure (at left) and predicted lattice rotations plotted as an OIM image (at right). Black boundaries indicate orientation changes of $>5^{\circ}$.

aluminum single crystals, two bicrystals were constructed identical (within the precision of the instruments) to the geometry used in the finite element simulation described above. Samples were characterized using OIM and the results are shown in Fig. 2. The long straight grain boundary is shown at center in each OIM image. Before the mechanical tests, the surfaces specified to remain unconstrained during deformation (i.e., the free plastic flow surface) were thoroughly characterized using OIM. From these measurements, microtexture information was extracted and the corresponding pole figures obtained. Detailed grain maps were also obtained by generating contrast between two grid points when the absolute angle of misorientation exceeded a specified amount. The two bicrystals were then deformed in channel-die compression to $10 \%$ and $30 \%$, respectively. Teflon tape was used as a lubrication to minimized local surface distortion. Following the deformation, the bicrystals were mapped for surface 

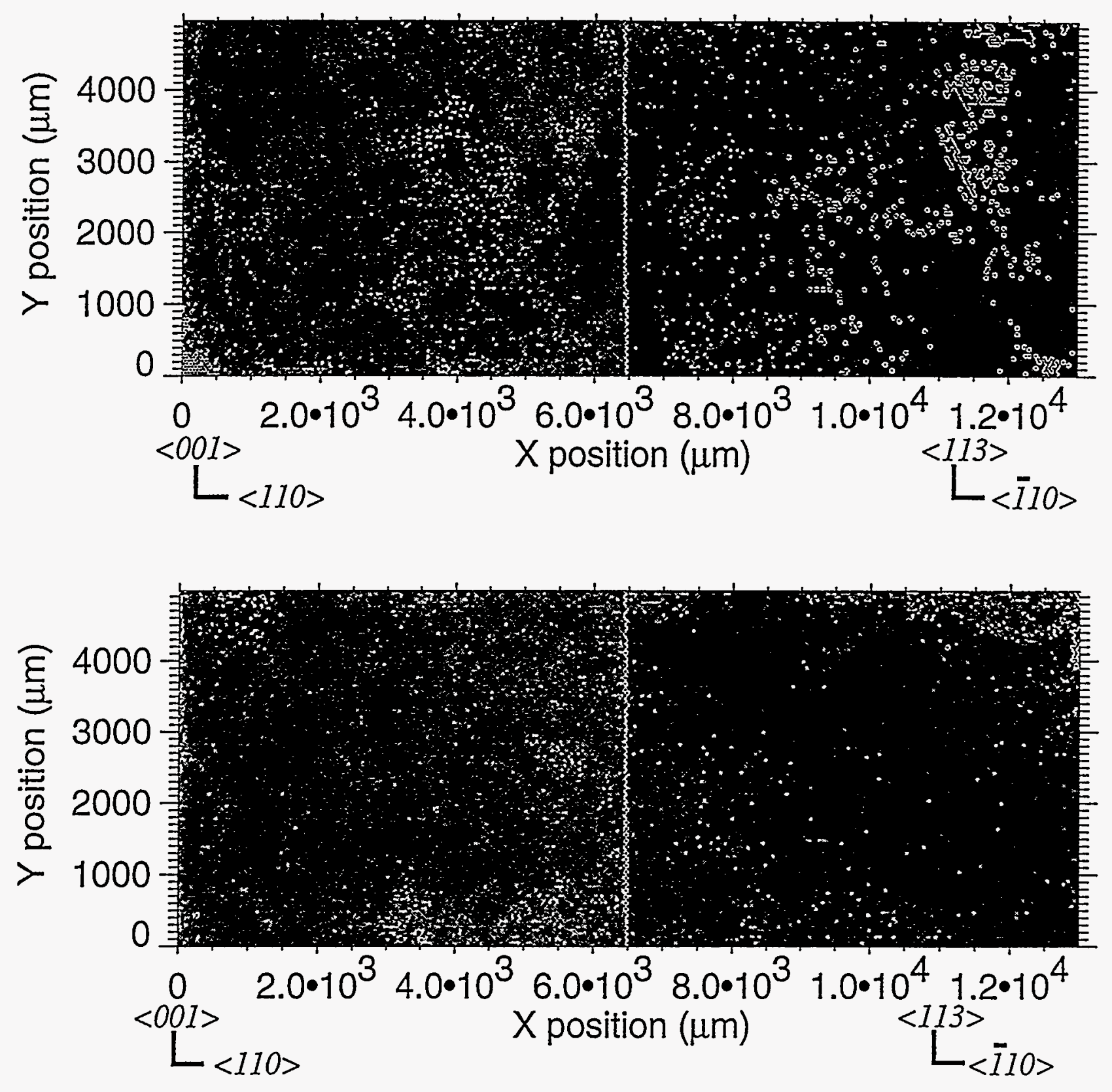

Figure 3. OIM images of samples prior to deformation. White lines indicate orientation changes of $>5^{\circ}$. Grain boundary is visible at center of each image. Relative orientations of crystals are indicated.

topography and surface strain, using optical measurements. The free surface of each sample was then mapped again using OIM to determine regions of substructure and localization (Fig. 3). From these experiments, pole figures and detailed grain maps were again produced (Fig. 4).

Comparison of the calculations with the experiments showed fair agreement for the bulk overall deformation response, e.g., the predicted response of the crystallite in the "harder" initial orientation showed less drastic localization and flow behavior then did the "softer" crystallite. However, the calculated response of the "softer" material was still stiffer than that showed by the experiment. Also, inspection of the predicted and measured pole figures showed reasonable agreement between the two 

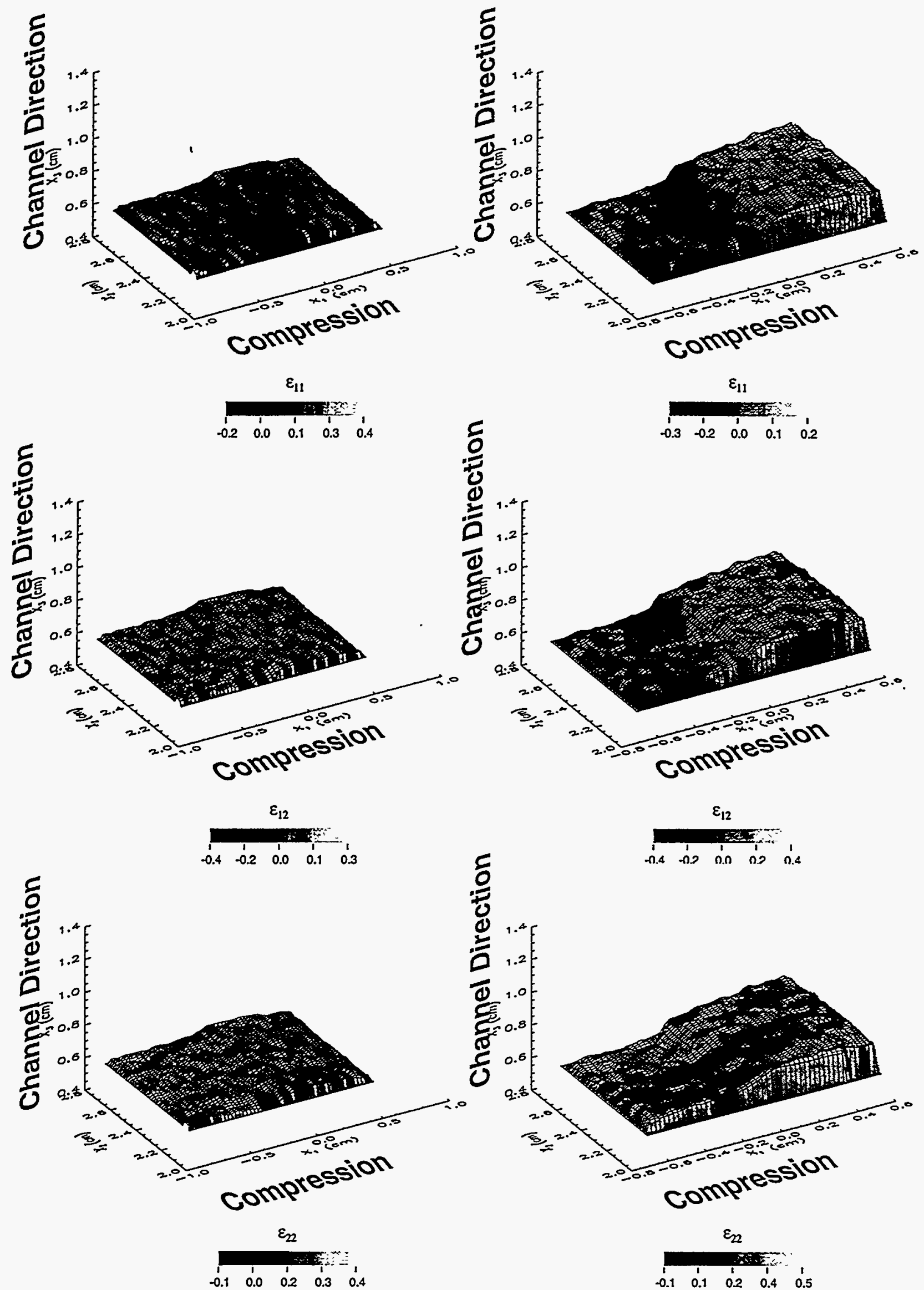

Figure 4. Surface topography and tensile strain in the compression direction (top), in-plane shear strain (center), and tensile strain in the transverse direction (bottom) plotted for $10 \%$ (left) and $30 \%$ (right) deformation of an aluminum bicrystal. 


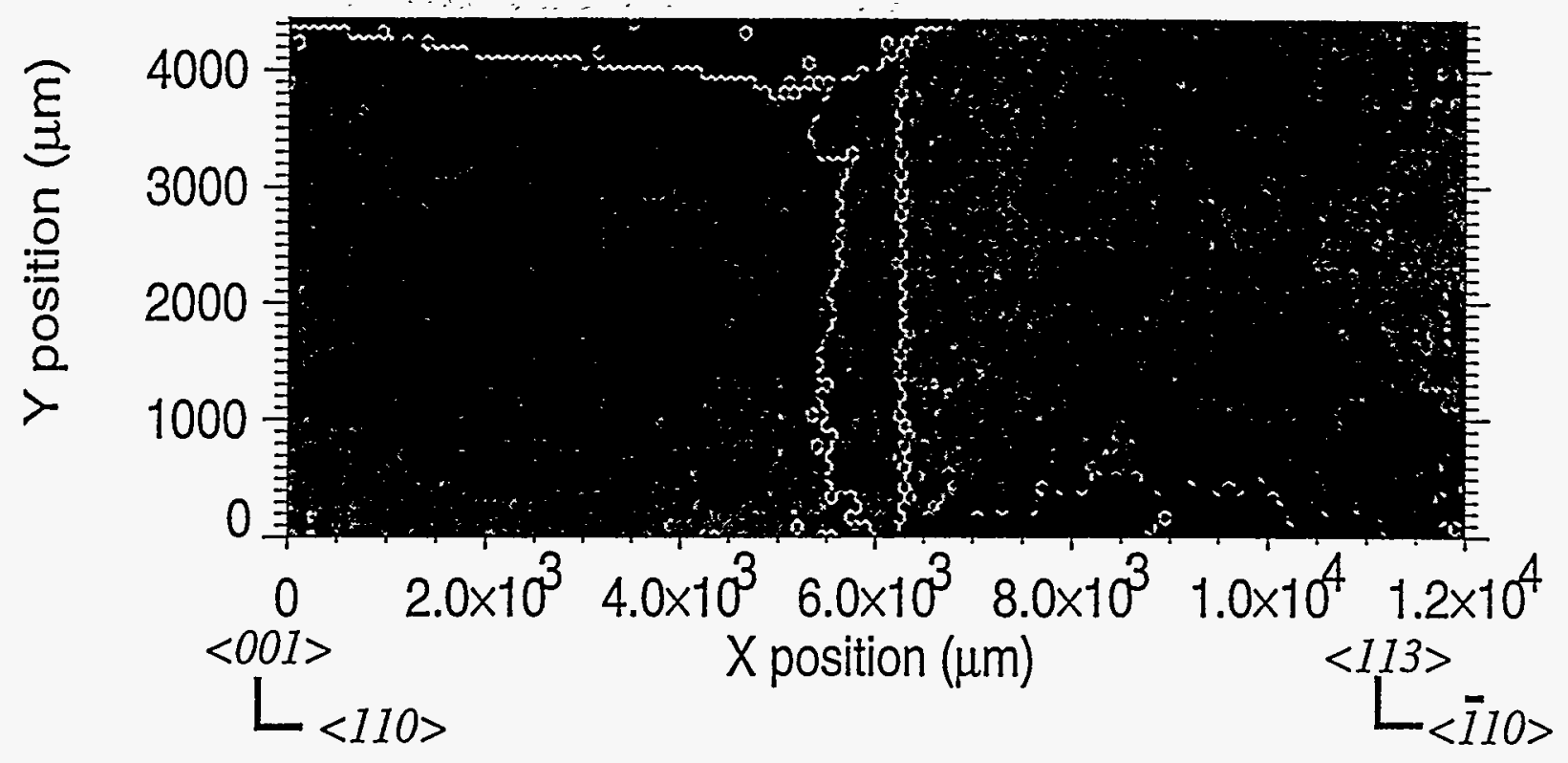

Figure 5 OIM image of $10 \%$ deformed bicrystal. White lines indicate orientation changes of $>5^{\circ}$.

\section{Compression Direction}

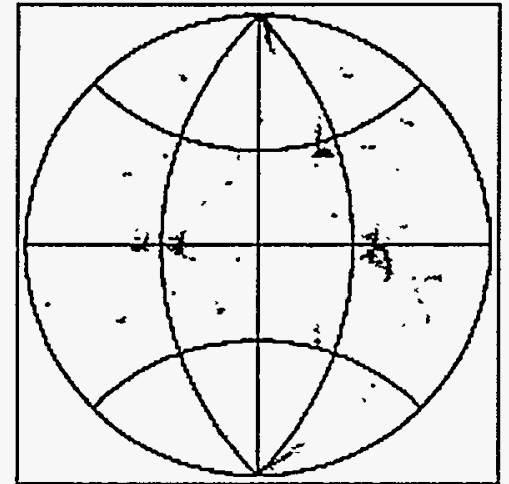

$10 \%$

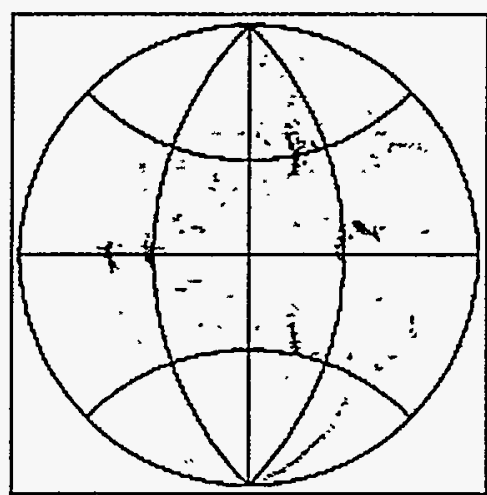

$30 \%$

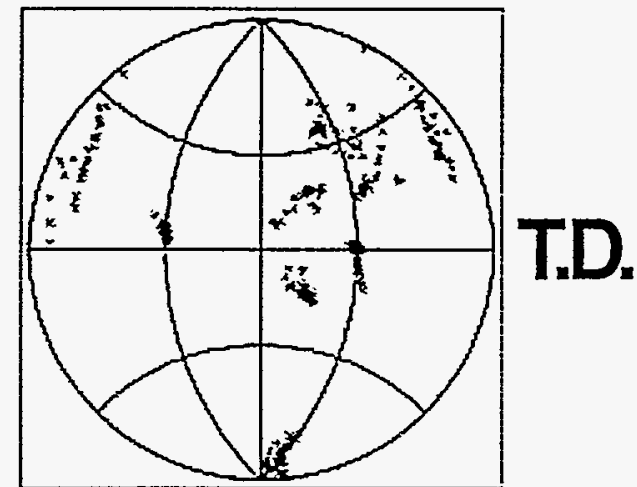

Simulated $30 \%$

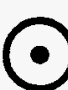

Channel Direction

Figure 6. Experimental pole figures after 10\% (left), 30\% (center) and simulated 30\% (right). 
in some of the stereological locations and poor agreement in other locations. Possible reasons for the lack of agreement include poor image quality from the OIM measurement due to the rough surface topology on the deformed surfaces and oxidation. Also, the finite element calculations used single point element integration that can result in a stiffer predicted response. Measures are currently under way to correct some of these problems: careful surface preparations have been performed, and higherorder integration schemes are being implemented in the simulations.

FUTURE WORK: This single crystal constitutive formulation includes no information about the length-scale of the material, geometry or deformation process. Recent work has shown strong correlations between plasticity phenomena (e.g., hardening) and material length-scale, and has suggested the need for more sophisticated plasticity laws ${ }^{5}$. Such a formulation, based on strain gradient theory, has been developed and is currently being demonstrated for microindentation predictions in isotropic materials ${ }^{6}$. The size effects that are demonstrated in microindentation tests can be predicted by the strain gradient theory. In the near future, work will include development of a new crystal plasticity model in the context of strain-gradient plasticity. Such a model will then be implemented in an implicit finite element code and demonstrated with microindentation calculations on a single crystal. Such calculations will be compared with the results from the conventional crystal-plastic model that was described aboye. Finally, the results from these calculations will be directly compared with microindentation experiments on single crystals of copper or aluminum. Correlated with these experiments, the analyses can be used to either confirm or rebut the strain gradient theory. Once a strain gradient theory is successfully implemented in a single crystal computational scheme, more sophisticated homogenization schemes, than are currently available in polycrystal calculations, will be needed which include misorientation and morphology of a microstructure. Work is currently under way to develop such homogenization theories.

\section{References}

${ }^{1}$ W. E. King, G. H. Campbell, A. W. Coombs et al., "Interface Science of Controlled Metal/Metal and Metal/Ceramic Interfaces Prepared using Ultrahigh Vacuum Diffusion Bonding," Mater. Res. Soc. Symp. Proc. 314, 61-67 (1993).

${ }^{2}$ B.L. Adams, S.I. Wright, and K. Kunze, "Orientation Imaging - The Emergence Of A New Microscopy," Metallurgical Transactions A-Physical Metallurgy And Materials Science 24 (4), 819-831 (1993).

${ }^{3}$ D. Peirce, R.J. Asaro, and A. Needleman, "Material Rate Dependence and Localized Deformation in Crystalline Solids," Acta Metallurgica Et Materialia 31, 1951 (1983).

${ }^{4} \mathrm{R}$. Becker, "Analysis Of Texture Evolution In Channel Die Compression .1. Effects Of Grain Interaction," Acta Metallurgica Et Materialia 39 (6), 1211-1230 (1991).

${ }^{5}$ N.A. Fleck, G.M. Muller, M.F. Ashby et al., "Strain Gradient Plasticity - Theory and Experiment," Acta Metallurgica et Materialia 42 (2), 475-487 (1994).

${ }^{6} J . Y$. Shu and N.A. Fleck, "Finite element analysis of indentation - the role of micro length scale,", (in preparation) (1996). 


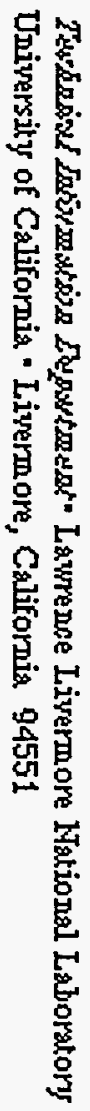

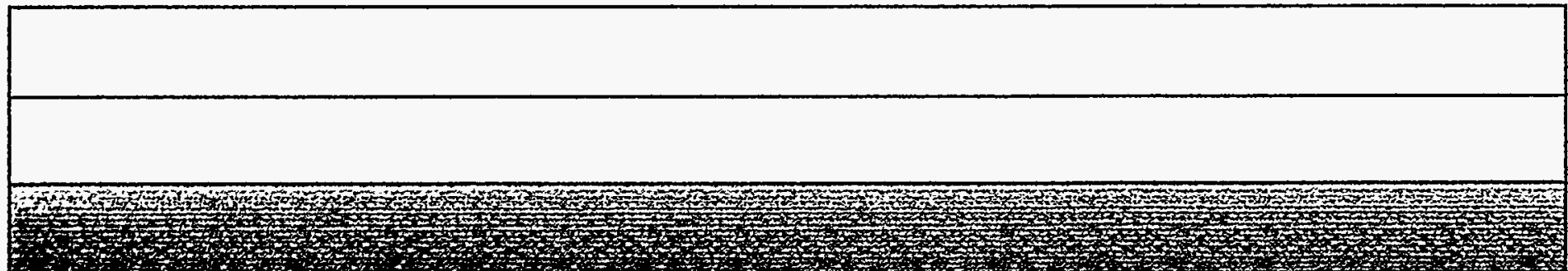

